\title{
Diffractive sidewall grating coupler: towards 2D free-space optics on chip.
}

\author{
A. Hadij-ElHouati*a , P. Cheben ${ }^{\text {b }}$, A. Ortega-Moñux ${ }^{\text {a }}$, J. G. Wangüemert-Pérez ${ }^{\mathrm{a}}$, R. Halir, J. H. \\ Schmid $^{\mathrm{b}}$, I. Molina-Fernández ${ }^{\mathrm{a}}$. \\ ${ }^{a}$ Universidad de Málaga, ETSI Telecomunicación, Campus de Teatinos s/n, 29071 Málaga, Spain \\ ${ }^{b}$ National Research Council of Canada, Ottawa K1A OR6, Canada
}

\begin{abstract}
Silicon photonics has been the subject of intense research efforts. In order to implement complex integrated silicon photonic devices and systems, a wide range of robust building blocks is needed. Waveguide couplers are fundamental devices in integrated optics, enabling different functionalities such as power dividers, spot-size converters, coherent hybrids and fiber-chip coupling interfaces, to name a few. In this work we propose a new type of nanophotonic coupler based on sidewall grating (SIGRA) concept. SIGRAs have been used in the Bragg regime, for filtering applications, as well as in the sub-wavelength regime in multimode interference (MMI) couplers. However, the use of SIGRAs in the radiation regime has been very limited. Specifically, a coarse wavelength division multiplexer was proposed and experimentally validated. In this work we study the use of SIGRAs in the diffractive regime as a mean to couple the light between a silicon wire waveguide mode and a continuum of slab waveguide modes. We also propose an original technique for designing SIGRA based couplers, enabling the synthesis of arbitrary radiation field profile by FloquetBloch analysis of individual diffracting elements while substantially alleviating computational load. Results are further validated by 3D FDTD simulations which confirm that the radiated field profile closely matches the target design field.
\end{abstract}

Keywords: sidewall grating, coupler, spot-size converter, sub-wavelength grating, Floquet-Bloch analysis, diffraction grating, silicon photonics, distributed Bragg deflector.

\section{INTRODUCTION}

Silicon photonics has emerged as a powerful platform for integrating thousands of components due to its high index contrast and compatibility with the CMOS process, which is well established in the microelectronics industry. As a result, it permits low cost chips in mass production environments since facilities and procedures are already developed and it also enables integration of electronics and photonics in the same platform ${ }^{1}$. Current electronic processors could take advantage of on-chip optical links to speed up communications between cores as this is a bottleneck ${ }^{2}$.

One shortcoming of the CMOS process is that it allows a limited set of materials (mainly silicon and silicon dioxide) with fixed refractive indexes. Metamaterials based in Sub-Wavelength Gratings (SWG) can partially circumvent this drawback by offering a path to synthetize the effective refractive index of the artificial material by simple means. SWGs are periodic structures with a period small compared to the wavelength, so that they behave as a homogeneous and anisotropic material whose optical properties can be engineered by proper design of their geometry ${ }^{3}$.

Applications of periodic structures in photonics go way beyond metamaterial synthesis. Periodic structures with pitch big enough compared to the wavelength called diffractive gratings are widely used to implement fiber-chip couplers ${ }^{4,5}$. Diffractive gratings are widely used for fiber-chip interface since they offer a simple and yet versatile access to the chip allowing wafer scale testing. Other type of periodic structures, Bragg gratings, are widely used to implement wavelength-selective devices ${ }^{6-8}$.

*abdel@uma.es 
Diffractive gratings usage in silicon photonics had been limited to fiber-chip couplers or vertical substrate to substrate coupling ${ }^{9}$. Few works have explored the usage of gratings in the diffractive regime for shaping the light without extracting it out of the chip plane. Firstly proposed by Stoll ${ }^{10}$, distributed Bragg deflectors (DBD) consist of a channel waveguide that is periodically perturbed to arise a controlled diffraction that is coupled into a one-dimensionally confined slab waveguide. It would be desirable to transfer DBD to the silicon photonics platforms, however, some adaptations must be done to the original idea. In this work we make use of a diffractive element similar to the one originally proposed for a wavelength demultiplexer ${ }^{11,12}$. Contrary to previous DBD designs, based in a shallow etch on top of the waveguide as done in Stoll ${ }^{10}$ or the small index perturbation in index implemented in Davis ${ }^{13}$, this design makes use of a strong blazing etch in the sidewall of a silicon wire waveguide to implement the grating. Besides, a SWG synthesized metamaterial is used to separate the waveguide from the free propagation region (FPR).

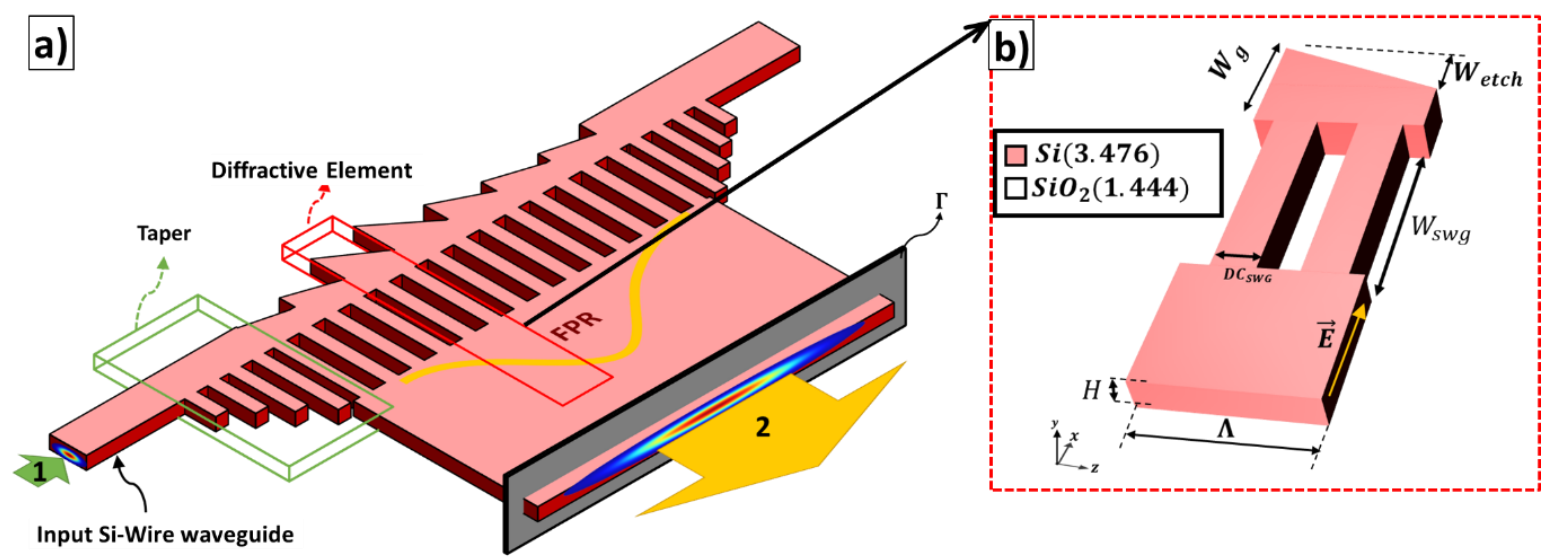

Figure 1. a) Simplified schematic of a SIGRA mode expander composed by succession of diffractive elements b), an input taper and a free propagation region (FPR).

One immediate application for the DBD SIGRA is to build a beam expander as compact as state of the art devices ${ }^{14}$. Beam expanders, devices that change the mode spot size, are fundamental to connect the wide fiber-chip couplers $(\sim 15 \mu \mathrm{m})$ to the narrow standard silicon wire waveguide $(500 \mathrm{~nm})$. The simplest and most straightforward way to implement this expansion is using linear tapers. However, this approach obtains extremely long devices $(\sim 150 \mu m)$ since a steep change in width would cause power transfer to undesired higher order modes. Extensive research has been made to obtain compact and efficient spot-size converters ${ }^{14}$. In this work we propose a new compact architecture for beam expansion in the SOI platform based on apodised SIGRA DBD that is shown in Figure 1. This device works as follows: light enters the device from a standard silicon wire guide (1), after that it encounters a taper that gradually introduces the SWG pillars of the metamaterial region. The main aim for this taper is to increase power to the fundamental SIGRA mode and decrease the power loss due to reflections and excitation of undesired higher order modes. As the lights propagates in the SIGRA it gets diffracted progressively. Ideally, the diffracted field should completely be captured into the Free Propagation Region (FPR). In order to enhance the power entering the FPR, a metamaterial implemented by SWG pillars is used.

SIGRA based beam expander could also be advantageous if used as a feeding element for Optical Phased Arrays (OPA), which are the natural extension to the optical domain of the arrays classically used in radiofrequency for addressing beam steering applications. OPAs have been the subject of great interest in recent years ${ }^{15}$ since they allow generating very narrow collimated optical beams and controlling its direction of emission. The applications of this type of technology are potentially vast and cover possibilities that include, from the establishment of reconfigurable networks of optical communications in free space, to applications of sensing and navigation based on Light Detection and Ranging (LIDAR), a technology that has a great interest for the emerging industry of autonomous vehicles. Figure 2 depicts a wide grating based antenna fed by a compact SIGRA coupler. As it will be seen in Section 3, the use of a SIGRA as the feeding element of a radiating grating allows the beam to be steered in the chip plane with wavelength. 


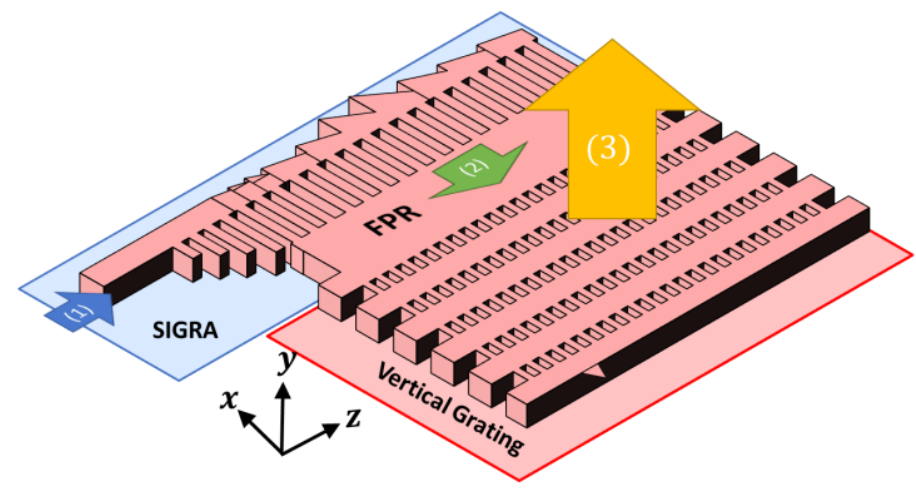

Figure 2. OPA formed by a SIGRA coupler that feeds a wide vertical coupler. Light entering from a standard si-wire waveguide (1) is fed to a SIGRA coupler that diffracts it towards the FPR as a Gaussian beam, then the bean enters the vertical grating that radiates it out of the chip (3).

What is more, a SIGRA coupler could be conveniently designed to generate a focusing Gaussian beam that is able to propagate in any direction in a one-dimensionally confined slab region. Another similarly designed SIGRA could be positioned in hundreds of microns apart to capture this beam and transform it again into a guided mode. In this way, the on-chip 2D wireless system could be implemented as depicted in Figure 3. This would allow reconfigurable light paths and low crosstalk crossings ${ }^{16}$. This would be a pyramidal building block to enable on-chip wireless communications.

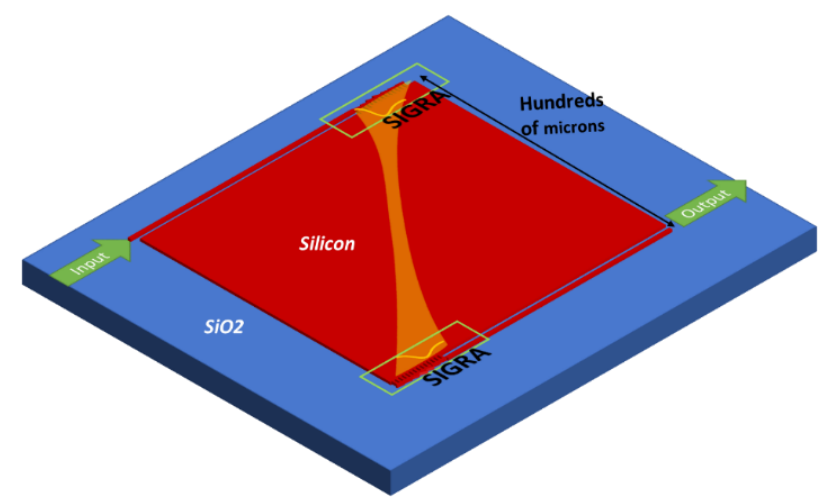

Figure 3. On-chip 2D free-space link based on two SIGRA couplers.

The specific objective of this study is to establish the viability of using SIGRA based DBD in the SOI platform and identify its advantages, opportunities and shortcomings. For that, a procedure for designing SIGRA devices based in Floquet-Bloch mode analysis is also proposed and two applications of SIGRA based DBD will be explored: beam expansion and OPA steering.

This document is structured as follows: in section 2 we discuss the details of SIGRA beam expander, in section 3 we analyze the usage of the SIGRA beam expander inside an OPA system, and finally in section 4 we discuss some conclusions and we suggest where future work should focus.

\section{DESIGN OF A SIGRA BEAM EXPANDER}

The design that follows was made for a Silicon-on-Insulator platform composed by a silicon layer of height of $220 \mathrm{~nm}$ surrounded by silicon dioxide. At the central wavelength of operation $\lambda=1550 \mathrm{~nm}$ the refractive indices of silicon and silicon dioxide are $n_{S i}=3.476$ and $n_{S_{i O}}=1.444$ respectively. The input waveguide width was set to $500 \mathrm{~nm}$ to ensure confinement and mono-mode operation. 


\subsection{SYSTEM LEVEL DESIGN}

The system purpose of the SIGRA coupler is to generate a Gaussian beam propagating inside the FPR slab. However, it is well known that an exactly periodic grating would produce an exponentially shaped profile which has a poor overlap with a Gaussian $(\sim 80 \%)$. Greater overlap efficiencies can be obtained through apodization. Nevertheless, a full apodization would require designing every diffractive element of the device resulting in a complex and slow process. In this work, we will use just two different diffractive elements, thus achieving a good trade-off between overlap and design complexity. Using two sections of two different diffractive elements results in a double exponential field profile given by:

$$
f(z)= \begin{cases}\mathrm{e}^{-\alpha_{\mathrm{a}} \mathrm{z}} & , 0<z<L \\ \sqrt{\frac{\alpha_{\mathrm{b}}}{\alpha_{a}}} \mathrm{e}^{-\alpha_{\mathrm{a}} L} \mathrm{e}^{-\alpha_{b}(\mathrm{z}-\mathrm{L})} & , z \geq L\end{cases}
$$

where $\alpha_{\mathrm{a}}$ and $\alpha_{\mathrm{b}}$ are the radiation strength of the first section and the second section respectively and $\mathrm{L}$ is the length of the first section. Optimization of the overlap of $f(z)$ with the targeted Gaussian profile with $40 \mu m$ mode-field radius (MFR) shows that $\alpha_{a}, \alpha_{b}$ and $L$ must be set to $0.005 \mathrm{~Np} / \mu \mathrm{m}, 0.0275 \mathrm{~Np} / \mu \mathrm{m}$ and $31.2 \mu \mathrm{m}$ respectively to reach the maximum overlap (92\%).

\subsection{DIFFRACTIVE ELEMENTS DESIGN}

Thus, now the design resumes to find the geometry parameters for the SIGRA diffracting elements that synthesize those radiation strengths. In general, proposed devices are optically long, thus design by brute force 3D analysis would be computationally demanding and it would take tremendous computation time. Consequently, a more judicious approach should be taken to efficiently design the full device. The approach, used in this work consists on individually characterizing the diffractive elements by Bloch-Floquet mode analysis ${ }^{17}$ to extract the electromagnetic parameters of interest, i.e. radiation strength $\alpha$, propagation angle in the FPR slab $\theta$ and efficiency. From this analysis complete design of the radiating structure can be performed. Once it is done, 3D FDTD analysis of the full device will be performed once, just to test that the final design goals have been achieved. In a SIGRA, efficiency is measured as the fraction of the input power that is coupled to the desired field profile inside the FPR region. Even using Bloch-Floquet mode analysis the design universe is still huge. Because of that, in a first phase, we decided to apply the effective index method (EIM) to transform the tridimensional structure into a $2 \mathrm{D}$ equivalent one. This will permit not only a faster simulation, but also an easier interpretation of the results. The design obtained by 2D Bloch-Floquet mode will be verified using a 3D BlochFloquet analysis and a refinement of the design using 3D Bloch-Floquet analysis will be done if required. So, now the 2D equivalent model is shown in Figure 4.

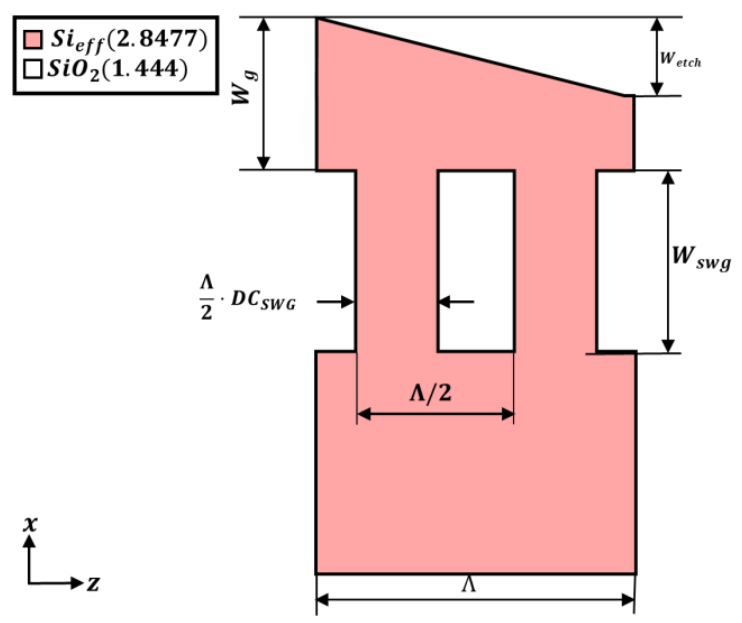

Figure 4. Diffractive element model after applying the EIM. 
Firstly, we designed the SWG region parameters $D C_{S W G}$ and $W_{S W G}$. In the step, we set the waveguide parameters to a typical value $\left(W_{g}=600 \mathrm{~nm}\right.$ and $\left.W_{\text {etch }}=300 \mathrm{~nm}\right)$. The subwavelength region duty cycle $D C_{S W G}$ was fixed to 0.5 to maximize the minimum feature size. Whereas, its width $W_{S W G}$ was set to $2 \mu \mathrm{m}$ to ensure that power loss due to leakage to zeroth diffraction order is below $10 \%$ of total diffracted power (see Figure 5) and yet keep the device compact.

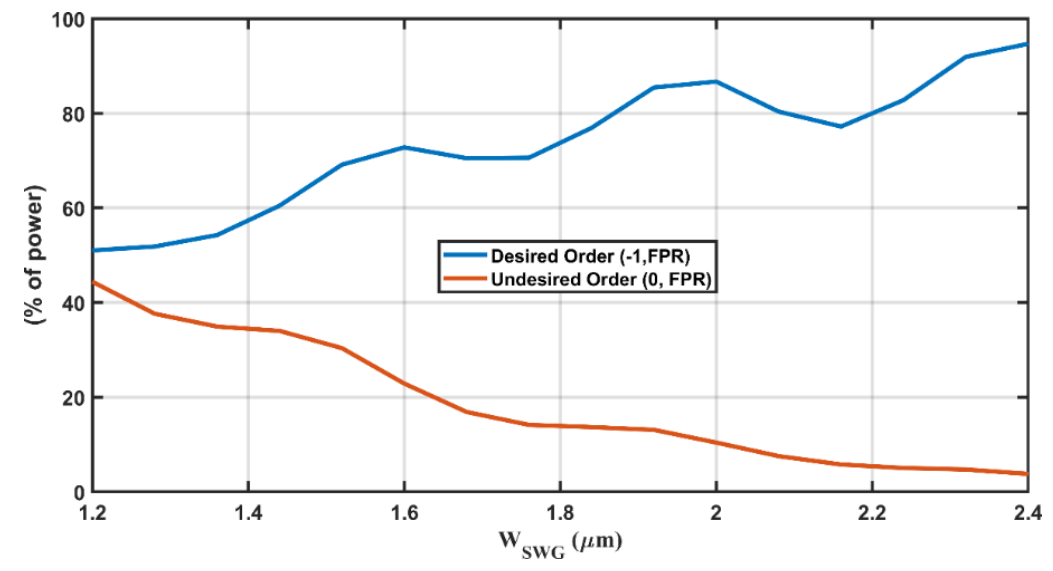

Figure 5. Radiated power distribution among different diffraction orders versus $W_{S W G}$.

Next, we designed $W_{g}$ and $W_{\text {etch }}$ together as these two parameters are tightly coupled and cannot be designed independently. In fact, for every $W_{g}$ we can find one and just one $W_{\text {etch }}$ that maximizes the efficiency as it can be seen in Figure 6. Thus, varying $W_{g}$ from $450 \mathrm{~nm}$ to $700 \mathrm{~nm}$ we can synthesize any radiation strength from $0.002 \mathrm{~Np} / \mu \mathrm{m}$ to $0.0275 \mathrm{~Np} / \mu \mathrm{m}$ with efficiencies ranging from $85 \%$ to $99 \%$.
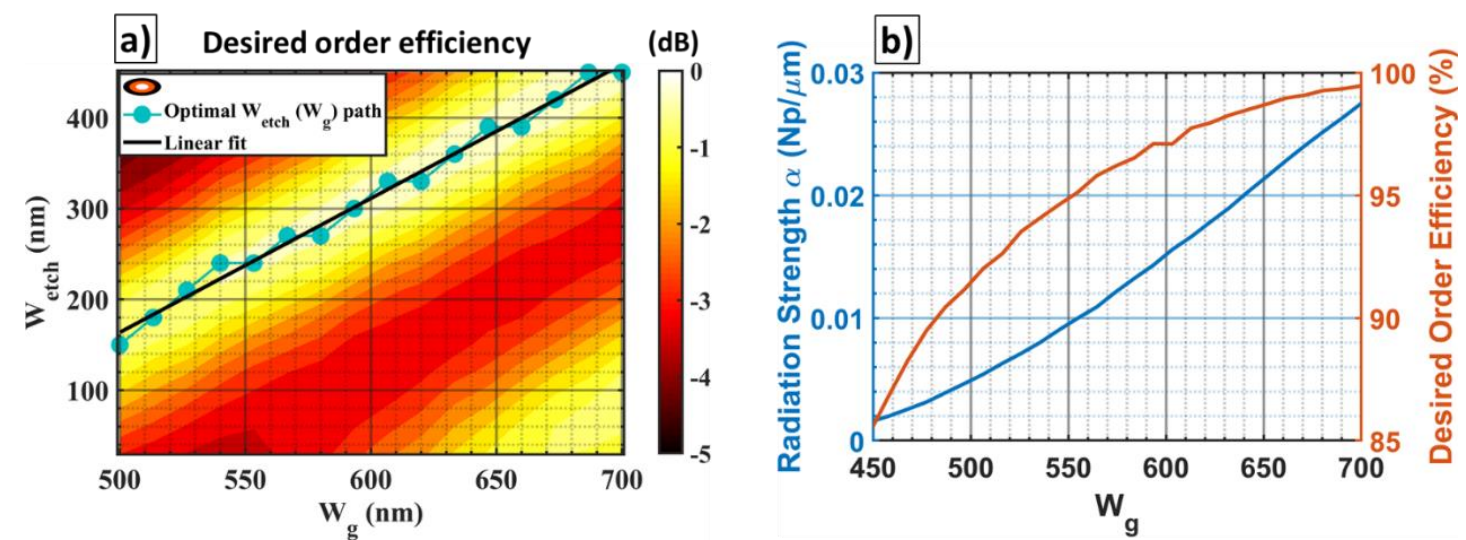

Figure 6.a) Desired order efficiency as function of $W_{g}$ and $W_{\text {etch }}$ and b) electromagnetic parameters along the fitted optimal path $W_{\text {etch }}=1.52 \cdot W_{g}-600 \mathrm{~nm}$.

In our case, to implement the desired double exponential field the geometries required are shown in Table 1.

Table 1. Geometric and electromagnetic parameters of the designed diffractive elements using a 2D Bloch-Floquet mode analysis.

\begin{tabular}{|c|c|c|c|c|c|c|c|c|}
\hline & $W_{g}(\mathbf{n m})$ & $W_{\text {etch }}(\mathbf{n m})$ & $W_{\text {SWG }}(\mu \mathrm{m})$ & $\Lambda(\mathbf{n m})$ & $L(\mu \mathrm{m})$ & $D C_{\mathrm{SWG}}$ & $\alpha\left(\frac{N p}{\mu m}\right)$ & Efficiency \\
\hline SECTION A & 500 & 160 & 2 & 551.6 & 32 & 0.5 & 0.005 & $91 \%$ \\
\hline SECTION B & 700 & 464 & 2 & 555.7 & 128 & 0.5 & 0.0275 & $99.5 \%$ \\
\hline
\end{tabular}




\subsection{INPUT TAPER DESIGN}

The input adaptation taper must be sufficiently long to ensure that most of the entering power goes to the first FloquetBloch mode and reflected power is kept low. For the purpose of designing this element the architecture shown in Figure 7 composed by a taper $N_{S W G}$ periods of the non-diffractive SWG guide that is formed when $W_{g}$ is fixed to $600 \mathrm{~nm}$ and $W_{\text {etch }}$ to $0 \mathrm{~nm}$. The transmission and reflection of this structure had been evaluated for various number of taper elements and for different period of the SWG region. Considering different SWG periods enable us to detract the interferometric effect related to the architecture. For 25 taper elements it is more than enough to achieve both negligible reflection (-30 $\mathrm{dB})$ and high transmission $(-0.05 \mathrm{~dB})$.
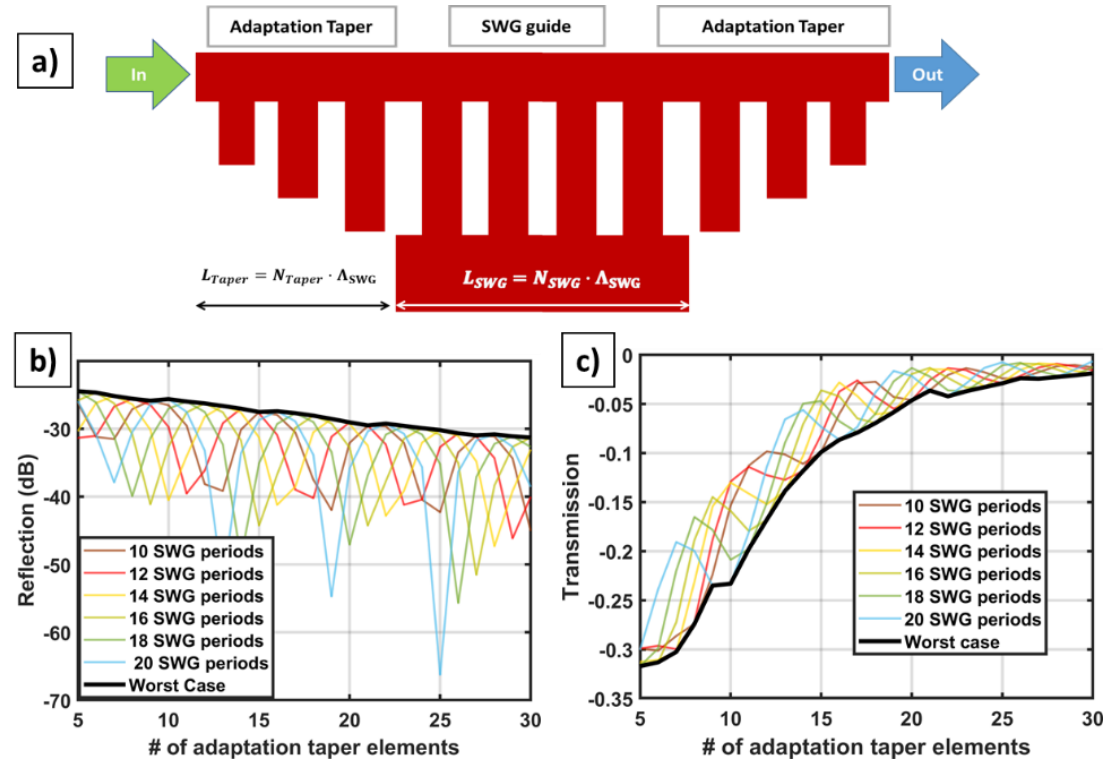

Figure 7. Architecture used to design the taper (a) and reflection (b) and transmission (c) of this architecture for various number of taper elements. It has been marked un bold black the worst case in both cases.

\subsection{FULL DEVICE VERIFICATION IN A 2D SIMULATOR}

To verify the design a FDTD simulation of the full structure consisting of the input and output tapers and the SIGRA was performed. The total efficiency obtained is $88.5 \%$, meaning that $88.5 \%$ of the power entering the input si-wire is transferred to a Gaussian of $40 \mu \mathrm{m}$ MFR. Most of the power is lost due to field mismatch since the double gaussian profile achieves $92 \%$ of overlap with the Gaussian. The rest of the lost power goes to undesired diffraction orders.
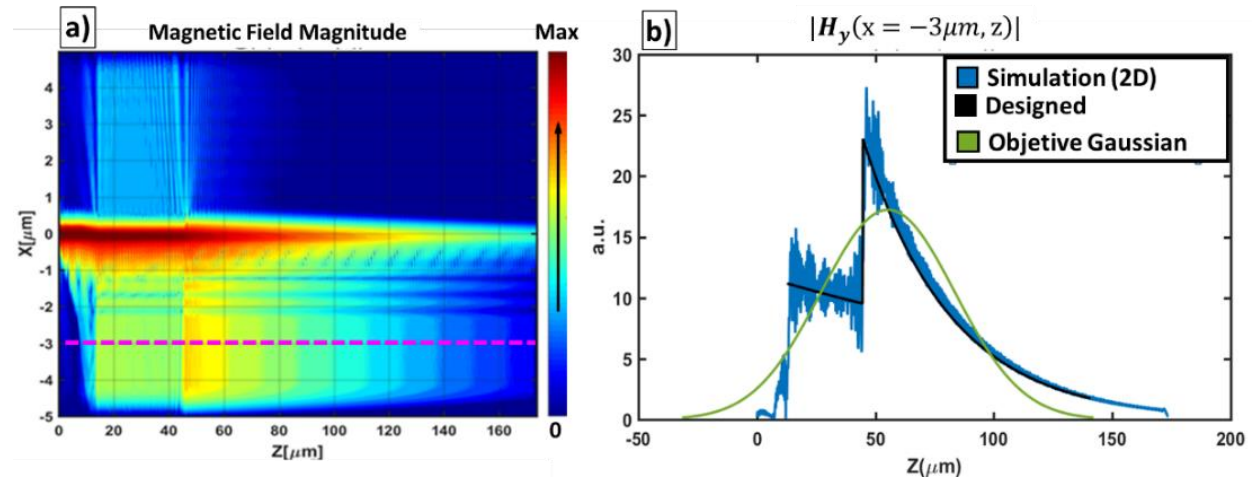

Figure 8. a) Magnetic field when the fundamental mode is injected in the input si-wire waveguide resulted from 2D simulation. b) Field profile magnitude along the cut indicated in pink line (blue) and comparison with the designed profile (black) and the aimed Gaussian profile of $M F R=40 \mu \mathrm{m}$. 


\subsection{VERIFICATION IN A 3D SIMULATOR}

3D Floquet-Bloch mode analysis of the designed element shows that there is a huge loss due to radiation out of chip unaccounted for in the 2D model. This can be seen in the field profile shown in Figure 9. What is more, the radiation strengths $\alpha_{a}$ and $\alpha_{b}$ are twice those predicted by the 2D model. This discrepancy translates in a reduction in the field profile overlap with the objective Gaussian. Thereby, a refinement process of the individual elements made by $3 \mathrm{D}$ Floquet-Bloch mode analysis carried on correcting the value of $\alpha_{a}$ and $\alpha_{b}$ and improving slightly the efficiency. After the refinement we obtained a new design whose parameters are shown in Table 2.
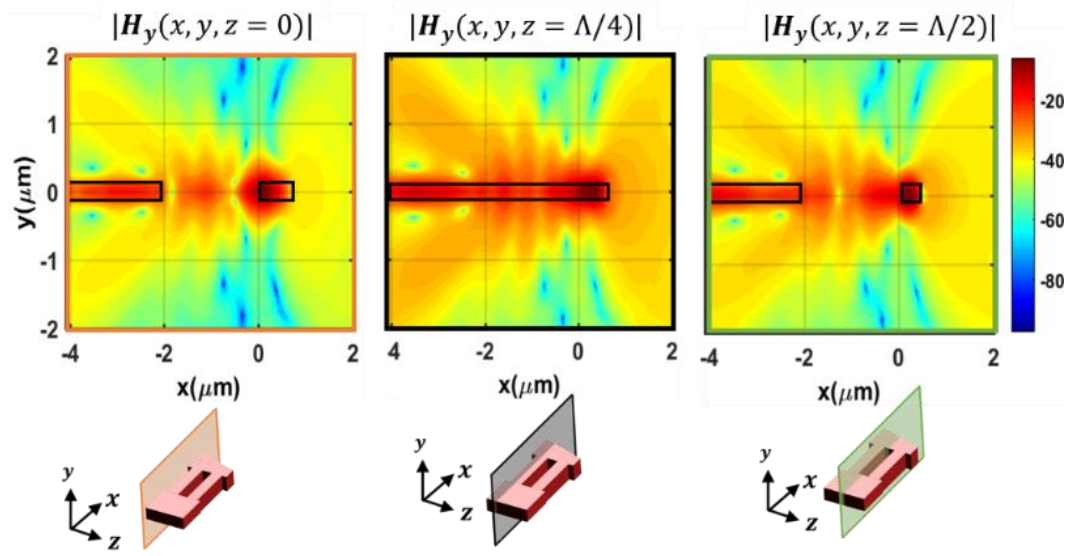

Figure 9.Various magnetic field cuts obtained by 3D Floquet-Bloch analysis showing the loss of power out of chip.

Table 2. Geometric and electromagnetic parameters of the designed diffractive elements using 3D Bloch-Floquet mode analysis.

\begin{tabular}{|c|c|c|c|c|c|c|c|c|}
\hline & $W_{g}(\mathrm{~nm})$ & $W_{\text {etch }}(\mathbf{n m})$ & $W_{\text {SWG }}(\mu \mathrm{m})$ & $\Lambda(\mathbf{n m})$ & $L(\mu \mathbf{m})$ & $D C_{\text {SWG }}$ & $\alpha\left(\frac{N p}{\mu m}\right)$ & Efficiency \\
\hline SECTION A & 500 & 110 & 3 & 551.4 & 32 & 0.5 & 0.005 & $51 \%$ \\
\hline SECTION B & 625 & 310 & 3 & 548.6 & 128 & 0.5 & 0.026 & $48 \%$ \\
\hline
\end{tabular}

Finally, operation of the complete structure in the bandwidth of interest $(\lambda=1.5 \mu \mathrm{m} \ldots 1.6 \mu \mathrm{m})$ is simulated. For this purpose, RSoft FULLWAVE FDTD $3 \mathrm{D}^{18}$ simulator has been used to calculate the resulting field profile at the FPR. Then, this field has been approximated as a Gaussian beam characterized by its modal radius (Figure 10.c), propagation direction (Figure 10.b) and normalized power (power relative to the input power) (Figure 10.a). From these figures it is evident that efficiency has drastically been reduced compared to the $2 \mathrm{D}$ case.
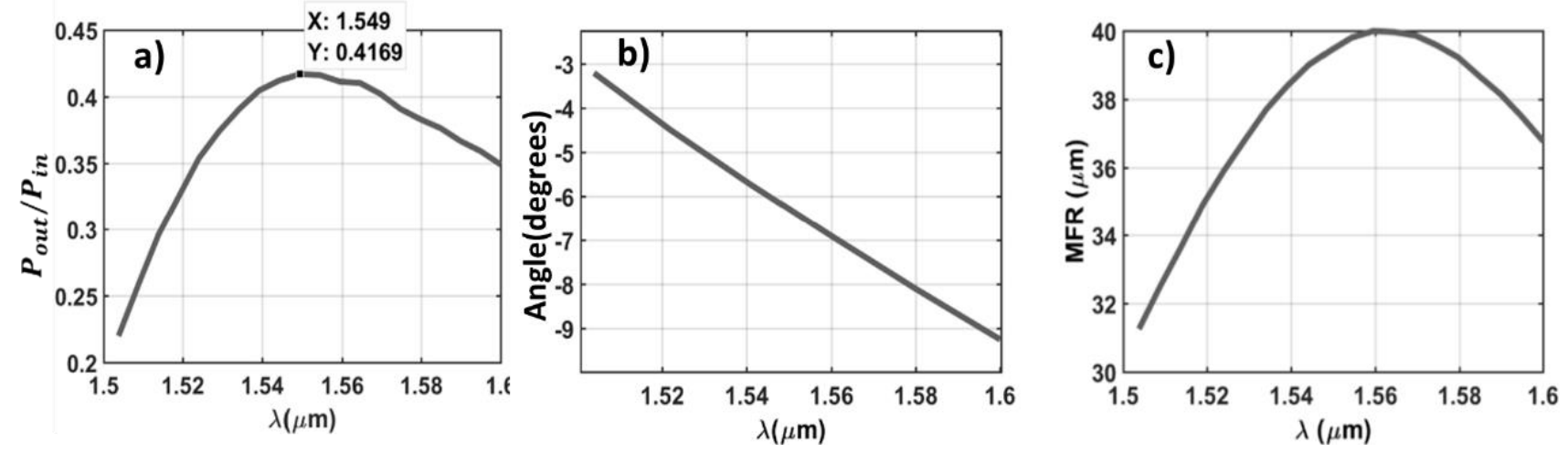

Figure 10. Characteristic values of the equivalent gaussian generated in the FPR slab. 


\section{SIGRA COUPLER USAGE IN A WAVELENGTH-STEERABLE OPA}

A wavelength steerable optical phased array was built by attaching the SIGRA beam expander designed in the previous section to an uniform $160 \mu m \times 160 \mu m$ rating coupler based on SWG structures based on the design of Halir ${ }^{5}$. The vertical grating was rotated slightly so that at central wavelength $(\lambda=1550 \mathrm{~nm})$ the beam generated by the SIGRA coupler incided normally. To analyze the functioning of this system we used the systemic parameters of the SIGRA coupler computed in the previous section to define the input excitation of the vertical grating in a 3D-FDTD simulator. The near field was extracted, and the far field was computed by computing bidimensional Fourier transform ${ }^{19\left(\text { chap }^{4}\right)}$. The radiated beam was found to have FWHM of $1^{\circ} \mathrm{x} 0.5^{\circ}$ and its direction varied simultaneously along the azimuth and elevation as the wavelength changes (see Figure 11). Vertical grating fixes the elevation angle $\theta$ whereas the azimuth is fixed by the SIGRA coupler.
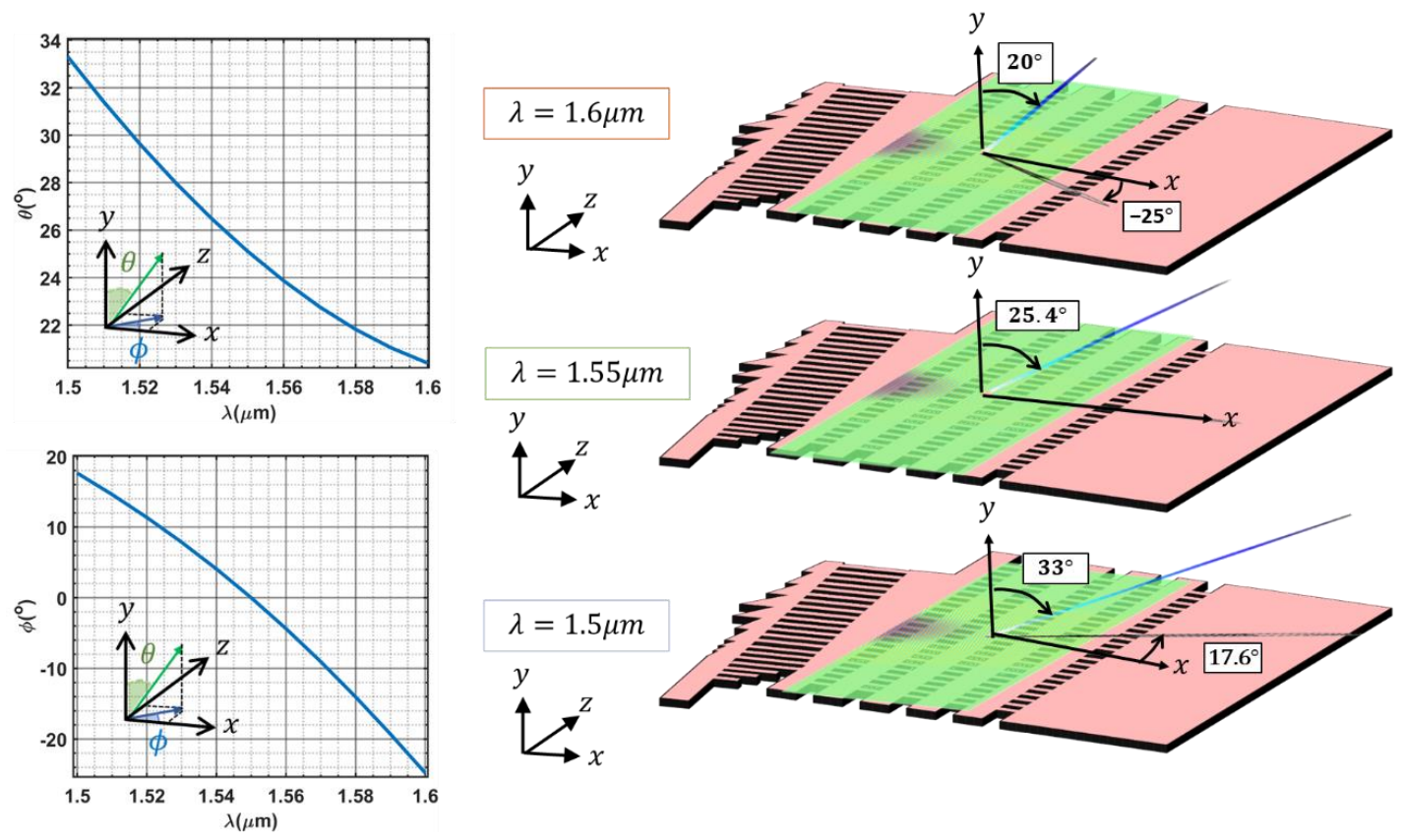

Figure 11. Elevation (a) and azimuth (b) of the radiated field as the wavelength varies. Near field on top of the system, geometry as well as far field for $\lambda=1.5 \mu \mathrm{m}$ (c) , $\lambda=1.55 \mu \mathrm{m}$ (d) and $\lambda=1.6 \mu \mathrm{m}$ (d)

\section{CONCLUSIONS}

In this work we have explored the basic functioning and some possible applications of the SIGRA coupler. In doing so, a new design technique, based on Bloch-Floquet mode analysis, has been proposed. A SIGRA coupler was designed using this technique. Preliminary simulation work was carried out by means of $2 \mathrm{D}$ simulation tool based in EIM, which allowed developing successful design strategies. Then rigorous 3D Floquet-Bloch mode simulation was used to completely design the device, and final 3D FDTD simulation of the complete device was used to check the device performance. Despite the high insertion losses of the device, due to out of plane radiation, the proposed strategy has shown to be a successful approach for designing this type of device. Work is in progress to find alternative approaches which will allow reducing the out of chip losses and thus will turn SIGRAs into a useful option for real applications.

\section{ACKNOWLEDGEMENTS}

We would like to acknowledge the Ministerio de Economía y Competitividad, Programa Estatal de Investigación, Desarrollo e Innovación Orientada a los Retos de la Sociedad (cofinanciado FEDER), Proyecto TEC2016-80718-R, the Ministerio de Educación, Cultura y Deporte (FPU16/03401), and the Universidad de Málaga. 


\section{REFERENCES}

[1] Chrostowski, L. and Hochberg, M., [Silicon Photonics Design], Cambridge University Press (2015).

[2] Sun, C., Wade, M. T., Lee, Y., Orcutt, J. S., Alloatti, L., Georgas, M. S., Waterman, A. S., Shainline, J. M., Avizienis, R. R., Lin, S., Moss, B. R., Kumar, R., Pavanello, F., Atabaki, A. H., Cook, H. M., Ou, A. J., Leu, J. C., Chen, Y.-H., Asanović, K., et al., "Single-chip microprocessor that communicates directly using light," Nature 528(7583), 534-538 (2015).

[3] Cheben, P., Halir, R., Schmid, J. H., Atwater, H. A. and Smith, D. R., "Subwavelength integrated photonics,” Nature 560(7720), 565-572 (2018).

[4] Benedikovic, D., Alonso-Ramos, C., Cheben, P., Schmid, J. H., Wang, S., Halir, R., Ortega-Moñux, A., Xu, D.-X., Vivien, L., Lapointe, J., Janz, S. and Dado, M., "Single-etch subwavelength engineered fiber-chip grating couplers for $13 \mu \mathrm{m}$ datacom wavelength band," Opt. Express 24(12), 12893 (2016).

[5] Halir, R., Cheben, P., Schmid, J. H., Ma, R., Bedard, D., Janz, S., Xu, D.-X., Densmore, A., Lapointe, J. and MolinaFernández, Í., "Continuously apodized fiber-to-chip surface grating coupler with refractive index engineered subwavelength structure," Opt. Lett. 35(19), 3243 (2010).

[6] Pérez-Galacho, D., Alonso-Ramos, C., Mazeas, F., Le Roux, X., Oser, D., Zhang, W., Marris-Morini, D., Labonté, L., Tanzilli, S., Cassan, É. and Vivien, L., "Optical pump-rejection filter based on silicon sub-wavelength engineered photonic structures," Opt. Lett. 42(8), 1468 (2017).

[7] Čtyroký, J., Gonzalo Wangüemert-Pérez, J., Kwiecien, P., Richter, I., Litvik, J., Schmid, J. H., Molina-Fernández, Í., Ortega-Moñux, A., Dado, M. and Cheben, P., "Design of narrowband Bragg spectral filters in subwavelength grating metamaterial waveguides," Opt. Express (2018).

[8] Briggs, R. M., Frez, C., Fradet, M., Forouhar, S., Blanchard, R., Diehl, L. and Pflügl, C., "Low-dissipation 74- $\mu$ m single-mode quantum cascade lasers without epitaxial regrowth," Opt. Express 24(13), 14589 (2016).

[9] Wan, C., Gaylord, T. K. and Bakir, M. S., "Grating design for interlayer optical interconnection of in-plane waveguides,” Appl. Opt. 55(10), 2601 (2016).

[10] Stoll, H. M., “Distributed Bragg deflector: a multifunctional integrated optical device,” Appl. Opt. 17(16), 2562 (1978).

[11] Bock, P. P. J., Cheben, P., Delâge, A., Schmid, J. J. H., Xu, D.-X., Janz, S. and Hall, T. J., "Demultiplexer with blazed waveguide sidewall grating and sub-wavelength grating structure," Opt. Express 20(18), 17616 (2008).

[12] Bock, P. J., Cheben, P., Schmid, J. H., Velasco, A. V, Delâge, A., Janz, S., Xu, D.-X., Lapointe, J., Hall, T. J. and Calvo, M. L., "Demonstration of a curved sidewall grating demultiplexer on silicon," Opt. Express 20(18), 1988219892 (2012).

[13] Davis, R. L., Long, W., Wang, C.-J., Lam, T., Ho, J. G., Nachman, P. M., Poylio, J., Mishechkin, O. V. and Fallahi, M., "Distributed Bragg Deflectors Fabricated in Sol-Gel Based Waveguides," IEEE Photonics Technol. Lett. 16(2), 464-466 (2004).

[14] Abbaslou, S., Gatdula, R., Lu, M., Stein, A. and Jiang, W., "Ultra-short beam expander with segmented curvature control: the emergence of a semi-lens," Opt. Lett. 42(21), 4383 (2017).

[15] Heck, M. J. R., "Highly integrated optical phased arrays: photonic integrated circuits for optical beam shaping and beam steering," Nanophotonics 6(1), 93-107 (2017).

[16] García-Meca, C., Lechago, S., Brimont, A., Griol, A., Mas, S., Sánchez, L., Bellieres, L., Losilla, N. S. and Martí, J., "On-chip wireless silicon photonics: from reconfigurable interconnects to lab-on-chip devices," Light Sci. Appl. 6(9), e17053 (2017).

[17] Joannopoulos, J. D., Johnson, S., Winn, J. N. J. and Meade, R. R. D., [Photonic crystals: molding the flow of light] (2008).

[18] RSoft., "RSoft FullWAVE."

[19] Saleh, B. E. A. and Teich, M. C., [Fundamentals of Photonics], Wiley, New York, USA (1997). 